\title{
Density and temperature controlled fluid extraction in a bacterial biofilm is determined by poly- $\gamma$-glutamic acid production
}

\author{
Ryan J. Morris ${ }^{\mathrm{a}}$, Tetyana Sukhodub ${ }^{\mathrm{b}}$, Cait E. MacPhee ${ }^{\mathrm{a}}$ and Nicola R. Stanley-Wall ${ }^{\mathrm{b}}$ \\ a National Biofilms Innovation Centre, School of Physics and Astronomy, The University of \\ Edinburgh, Edinburgh EH9 3FD, United Kingdom; ${ }^{\text {b}}$ Division of Molecular Microbiology, \\ School of Life Sciences, University of Dundee, Dundee DD1 5EH, United Kingdom
}

\begin{abstract}
A hallmark of microbial biofilms is the self-production of extracellular matrix that encases the cells resident within the community. The matrix provides protection from the environment, while spatial heterogeneity of expression influences the structural morphology and colony spreading dynamics. Bacillus subtilis is a model bacterial system used to uncover the regulatory pathways and key building blocks required for biofilm growth and development. Previous reports have suggested that poly- $\gamma$ glutamic acid (PGA) production is suppressed during biofilm formation and does not play a major role in biofilm morphology of the undomesticated isolate NCIB 3610. In this work we report on the observation of multiple travelling fronts that develop during the early stage of $B$. subtilis colony biofilm formation. We find the emergence of a highly motile population of bacteria that is facilitated by the extraction of fluid from the underlying agar substrate. Motility develops behind a moving front of fluid that propagates from the boundary of the biofilm towards the interior. The extent of proliferation is strongly modulated by the presence of extracellular polysaccharides (EPS). We trace the origin of this moving front of fluid to the production of PGA. We find that PGA production is correlated with higher temperatures, resulting in a mature biofilm morphology that is distinct from the biofilm architecture typically associated with B. subtilis. Our results suggest that B. subtilis NCIB 3610 produces distinct biofilm matrices in response to environmental conditions.
\end{abstract}

\section{KEYWORDS}

biofilms; polyglutamic acid; bacterial dynamics; Bacillus subtilis

\section{Introduction}

A common strategy employed by bacteria to mitigate stresses imposed by their environment is to co-exist in sessile communities known as biofilms. The transition from unicellular to multi-cellular life allows the residents to coordinate response to stimuli, share metabolic burdens ${ }^{1}$, and protect against external attack by predators ${ }^{2,3}$ or antimicrobial agents ${ }^{4,5}$. This behaviour is ubiquitous across the microbial world and a clear understanding of biofilm genesis, development, and maturation is important not only from a fundamental microbiological perspective but also due to their impact on many industrial, clinical, and biotechnological sectors. For example, biofilms act as sources for many chronic infections and their physical characteristics make them difficult to eradicate ${ }^{6,7}$. This intransigence can also impact industrial processes, where biofilms may result in pipe blockages, induce corrosion, or contaminate products ${ }^{8-10}$. While there are many negative consequences of biofilm formation, they play important 
roles in waste water treatment and other bioremediation processes ${ }^{11-14}$.

Bacillus subtilis is a Gram-positive bacterium that has been extensively used as a model organism to illuminate the genetic regulation and molecular mechanisms of biofilm formation ${ }^{15}$. During biofilm formation, production of the extracellular matrix is an essential process that binds the cells together, provides protection from the external environment, and confers advantageous mechanical properties to the community. The primary components of the matrix produced by $B$. subtilis are the fibrillar protein TasA ${ }^{16}$, the hydrophobin-like protein surfactant BslA ${ }^{17-19}$, and polysaccharides synthesized by products of the epsA-O operon ${ }^{20}$. One of the principle regulatory pathways that controls the expression of these components is modulated by the transcription factor Spo0A. Moderate levels of phosphorylated Spo0A activates transcription of the $\sin I-\sin R$ operon $^{21,22}$. SinR is a DNA-binding transcription factor that controls matrix production by interacting with the epsA-O and the tap $A$-sip $W$-tas $A$ promoters ${ }^{23}$. When SinR binds to its antagonist proteins (SinI and SlrR), repression is alleviated from these operons and biofilm formation can proceed ${ }^{24,25}$.

B. subtilis has several modes of active motility, two of which, swimming and swarming, are driven by the action of rotating flagella. Importantly, it has been shown that flagella-driven motility and biofilm phenotypes are bistable: cells can only express genes for motility or biofilm formation but not both at one time ${ }^{26,27}$. Bi-stability allows for the emergence of phenotypic heterogeneity within a population of genetically identical cells. ${ }^{28}$. This genetic bet-hedging provides a contingency for the community if and when environmental conditions change from their current state ${ }^{28,29}$.

In this work we report on the observation of multiple travelling fluid fronts that develop during the early stage of $B$. subtilis colony biofilm formation. We find the emergence of a highly motile population of bacteria that is facilitated by the extraction of fluid from the underlying agar substrate. Motility develops behind a moving front of fluid that propagates from the boundary of the colony towards the interior, and the extent of proliferation is modulated by a specific biofilm matrix component, the extracellular polysaccharide (EPS). We trace the origin of this moving front of fluid to the production of the polymer poly- $\gamma$-glutamic acid (PGA). We find the influx of fluid is dependent upon both bacterial density and environmental temperature. The temperature dependent production of PGA and the concomitant extraction of fluid can significantly impact the mature biofilm morphology, which diverges from the typical structure associated with $B$. subtilis NCIB 3610. Our results suggest that B. subtilis has the ability to produce an alternative extracellular matrix in response to adverse environmental conditions.

\section{Results}

\section{Deposition and imaging of growing biofilms}

At the beginning of each experiment, a $3 \mu \mathrm{L}$ suspension of $B$. subtilis cells $\left(\mathrm{OD}_{600}=\right.$ 1) are deposited onto MSgg agar, a biofilm-promoting minimal media ${ }^{30}$. After inoculation the droplet evaporates, which results in a 'coffee ring' deposition pattern: a higher density ring of bacteria accumulates at the edge of the initial droplet while the interior is more sparsely populated (Fig. 1A). This is caused by the differential rate of evaporation across the droplet; fluid evaporates more rapidly from the edge relative to the interior. This process drives capillary flows that transport bacteria from the interior to the boundary between the droplet and solid agar ${ }^{31}$. Our initial experiments 
were performed using the wild-type isolate NCIB 3610 that was allowed to grow at $38^{\circ} \mathrm{C}$ while time-resolved images were collected. The images were captured by imaging through the agar substrate and we monitor the dynamics of growth on the underside of the biofilm (Fig. 1A). We acquired images for the initial $\sim 6$ hours of growth. Examples of images taken in this manner are seen in Fig. 1B-D. Here, the high density 'coffee ring' region is clearly identifiable by the large accumulation of bacteria near the outer boundary of the colony (Fig. 1B). This high density region of bacteria often appears to be multi-layered with a width typically ranging from $75-100 \mu \mathrm{m}$.

\section{Emergence of a fluid flux that induces motility within the early biofilm}

After this initial time we observe a zone, emerging from the high density region, that is visually different to the interior of the colony (c.f. the dark annulus at the edge compared to the lighter interior, Fig. 1C). This zone grows and concurrently moves inwards as a travelling front towards the interior of the colony (Movie S1). Upon closer inspection of the travelling front we observe a highly dense and motile population of bacteria. The motile cells in this confined region clearly exhibit coherent patterns of self-organised motion such as swirls and vortices, reminiscent of structures observed in active turbulent systems (Movie S2) ${ }^{32,33}$. This behaviour implies that the cells are now in a fluid environment. The motility continues as this fluid zone grows, after which the motion rapidly stops (Fig. 1C) after travelling a distance of $\approx 400 \mu \mathrm{m}$ (Fig. 1D). This cessation of motility often also occurs as a propagating front, albeit travelling much faster than the initial propagation of motility (Movie S1, Movie S3) and predominantly moving from the interior of the colony back towards the coffee ring. Taken together, we infer that this moving front of motile cells represents a fluid flux into the biofilm.

Next, we added $2 \mu \mathrm{m}$ fluorescent beads to the suspension of bacteria that were deposited at the beginning of the experiment (Fig. 1E). Tracking the motion of the beads revealed that the beads coincident with the frontal zone are pushed along at a constant speed of $\approx 2.5 \mu \mathrm{m} \mathrm{min}^{-1}$ (Fig. $1 \mathrm{~F}$ ). Thus, the motion of this front has the ability to displace and mechanically push beads, but also cells, towards the center of the colony. A considerable portion of the beads remains embedded within the bacterial mass, but some become erratic in their movement. Our interpretation of this behaviour is that these beads are set in motion by the swimming action of the bacteria in a fluid environment. Interestingly, when we look at bead movement across the entire diameter of a colony, we also observe beads that move outwards radially from localized regions and this occurs simultaneously with the bead movement we see at the biofilm edge (Movie S4). Taking all data together, we infer that this moving front represents a fluid flux into the biofilm, and its emergence depends upon a high cell density.

\section{Extracellular polysaccharide production controls the spatial extent of the fluid flux}

Since we observe the formation of a fluid annulus at the outer edge of the biofilm, this led us to investigate if the extracellular matrix controls and modulates the extent of the fluid flux. We hypothesise that the formation of an annulus is a result of extracellular matrix production within the central region of the biofilm that prevents further incursion of fluid. We performed the same experiment described in Fig. 1A using a strain that possesses a deletion of $\sin R$, a key repressor of biofilm formation. Without $\sin R$, the cells over-produce the extracellular matrix and the colony biofilm occupies 
a smaller footprint and is highly wrinkled (Fig. S1) ${ }^{23}$. In this case, we posit that the fluid annulus should be more confined if the matrix impacts the spatial extent of fluid flow into the biofilm. We measured the maximum distance that the fluid encroached into the interior of the biofilm relative to the outer edge of the colony (Fig. S2, Movie $\mathrm{S} 5)$. We found that the distance the fluid travelled in the $\sin R$ strain was approximately 3 times less, on average, than the wild-type 3610 strain (Fig. 2). We conclude that extracellular matrix production can modulate the spatial extent of the fluid flux into the biofilm.

Next, we wished to determine if one or more individual components of the matrix were important in controlling the fluid flux. We performed analogous experiments on strains that possessed gene deletions for BslA (bslA), TasA (tasA), and EPS (epsA$O)$, respectively and we again measured the distance of fluid travel. We found that the tas $A$ and $b s l A$ strains were similar to the wild-type strain, albeit the fluid flux in the tas $A$ strain was slightly less than the wild-type and $b s l A$ strains (Fig. 2, Fig. S2). In contrast, the strain that produced the greatest difference in behaviour compared to NCIB 3610 was the strain that does not produce EPS. In this strain, as the fluid propagates inwards it is not confined to an annulus but moves to the very center of the colony as evidenced by the very large fluid travel distance (Fig. 2, Movies S1, S6, S7). This fluid coverage resulted in motile cells being visible across the entirety of the colony. Again, the motility was highly dynamic and we observed vortex formation characteristic of active turbulence (Movie S6). The motility arrest front is even more apparent in this strain and, like the fluid propagation front, travels across the entirety of the colony (Movie S1, S7, S8). While the tasA and bslA strains showed very similar dynamics to the wild-type in the first 6-8 hours, the mature biofilms of these matrix mutants resulted in smaller and more unstructured colonies compared to the wild-type strain (Fig. S1). Additionally, the epsA-O mature biofilms were similarly small and morphologically unstructured (Fig. S1) implying under these conditions, the initial fluid flux does not drastically perturb the colony morphology, and matrix production is the important driver in determining mature biofilm structure. Taken together our data demonstrate that the EPS is the determinant that controls the degree by which the fluid flux invades the biofilm.

\section{Whole biofilm imaging of epsA-O strain}

The pronounced fluid expansion and motility in the epsA-O strain allowed us to track the flux of fluid across the entirety of the biofilm in a time-resolved manner to learn more about the fluid extraction process. We noticed a finger-like instability develops as the fluid propagates inwards (Fig. 3A; Movies S7,S8). As the fluid pushes in towards the center of the colony the fluid front becomes unstable, forming increasingly large fingers over time (Fig. 3A,B). Such finger-like instabilities are well established in fluid dynamics. A well-known example is the Saffman-Taylor instability (also known as viscous fingering) that occurs when an unstable interface develops between a less viscous fluid displacing a fluid of higher viscosity ${ }^{34}$. Another way in which fingering morphologies can develop is through gradients in surface tension: Marangoni flows are induced when a fluid with lower surface tension flows towards regions of higher surface tension. Under appropriate conditions, a finger-like morphology can develop when an aqueous surfactant solution spreads on a thin-film of water ${ }^{35,36}$.

Imaging the biofilms in this manner also allowed us to fully track three key features of the dynamics: (1) the distance travelled by the expanding outer edge of the 
colony, (2) the distance travelled by the fluid flux, and (3) the motility arrest front. All measurements were taken relative to the initial position of each feature. Additionally, the relative distances were normalized by their maximum value to allow comparison of their temporal evolution (Fig. 3B). Initially, there is a lag time before the outer edge of the colony begins to expand at a constant rate beyond the initial deposition position. We find that the outer edge growth slows down and plateaus at precisely the time that we see the fluid front begin to propagate. Moreover, the onset of the arrest front propagation directly coincides with the resumption of growth at the outer edge of the biofilm.

\section{Polyglutamic acid is the agent that induces the fluid flux into the biofilm}

We wished to identify the molecule responsible for driving the fluid flux and subsequent motility and growth dynamics. Surfactin is a lipopeptide produced by B. subtilis that is a powerful biosurfactant ${ }^{37}$ and a potent anti-microbial agent ${ }^{38}$. Extracellular surfactin aids swarming and surface motility by lowering the surface tension of the fluid film and increasing the wettability of the substrate ${ }^{39,40}$, as well as acting as a 'oneway' signalling molecule that induces the activation of extracellular matrix genes ${ }^{41}$. Surfactin production in B. subtilis biofilms facilitates colony spreading ${ }^{40}$ and is important in the osmotic extraction of fluid from the underlying agar substrate ${ }^{42}$. Given these properties, we tested whether surfactin was the causative agent of the dynamics we observe. We used the srfAA and srfAA epsA-O deletion strains to compare the dynamics in both wild-type and EPS deficient backgrounds when surfactin could not be made. As a diagnostic for potential differences between the strains we again measure the relative displacement of the biofilm edge as a function of time. If the interior of the growing biofilm becomes fluidized we should see a plateau in the displacement curve as we saw in Fig. 3C. We found that when we measured the outer edge displacement for biofilms possessing these mutations, they both exhibited the characteristic 'fluidization plateau' (Fig. 4A). These data indicate that the srfA and srfA epsA-O strains did not abolish the fluid flux into the colony and led us to conclude that surfactin is not the causative agent of the fluid extraction.

Poly- $\gamma$-glutamic acid is a naturally occurring biopolymer consisting of repeating units of L-glutamic acid, D-glutamic acid or both, and is primarily produced by species of Bacillus ${ }^{43,44}$. PGA is another potential candidate due to its humectant properties $^{45,46}$ and its density dependent production ${ }^{47}$. To test whether PGA was responsible for the fluid extraction and the characteristic growth curves, we produced two deletion strains that targeted pgs $B$, the gene encoding the essential synthetase required for PGA production ${ }^{48}$. One strain possessed a deletion of $p g s B$, while the other possessed a deletion of the gene $p g s B$ in the eps $A-O$ background. Again, we measured the edge displacement simultaneously for the wild-type, epsA-O, pgsB, and pgsB epsA-O strains. We observe a plateau in the edge displacement for the wild-type and epsA-O strains while we observe no change in the displacement rate after the lag time for the pgs $B$ mutants (Fig. 4A). We also imaged the biofilm morphology after 48 hours of incubation and found that the wild-type and $p g s B$ mutant were morphologically similar (Figs. 4B,C), and different to the strains unable to produce EPS which were likewise comparable (Figs. 4E,F). For completeness, we also imaged the surfactin mutant biofilms and found that the $\operatorname{srfA}$ strain was structured but occupied a small footprint while the double $\operatorname{srfA}$ pgsB mutant was less structured, similar to the other non-EPS producing strains (Figs. 4D,G). We therefore conclude that PGA is the molecular agent 
that extracts fluid from the substrate and drives the dynamics that we have thus far described. Moreover, the initial fluid flux into the biofilm does not appreciably alter the morphology, and matrix production still governs the structural phenotypes of the colonies.

\section{$P G A$ expression is correlated with high temperature conditions}

The discovery that PGA was involved in biofilm formation was unexpected, as while PGA has a role in biofilm formation in several isolates of $B$. subtilis ${ }^{49}$ it had previously been excluded as a matrix molecule for NCIB $3610^{50}$. Its absence was reported to have no impact on colony biofilm structure ${ }^{47,50}$. We wanted to reconcile these divergent observations. We noted that our analysis was performed at $38^{\circ} \mathrm{C}$ while most other studies investigating $B$. subtilis biofilm formation are conducted at temperatures ranging from room temperature up to $30^{\circ} \mathrm{C}$. We therefore hypothesised that PGA production is temperature-dependent. First, we repeated the experiments with wild-type, eps $A-O, p g s B$, and pgsB epsA-O strains at the additional temperatures of $30^{\circ} \mathrm{C}$ and $42^{\circ} \mathrm{C}$ (the highest temperature achievable in our microscopy incubator). At $30^{\circ} \mathrm{C}$ we did not observe any fluid flow into the colony (i.e. no front development and motility). Likewise, there was no characteristic plateau in the edge displacement curve for all strains, even the wild-type and epsA-O strains (Fig. 5A). This result implies that PGA is not produced at $30^{\circ} \mathrm{C}$. At the higher temperatures of $38^{\circ} \mathrm{C}$, and $42^{\circ} \mathrm{C}$ we do find a plateau in the edge displacement curves for the strains able to produce PGA, while no plateau is observed for the PGA deficient mutants (Fig. 4A and Fig. 5B).

Second, NCIB 3610 was found to form exceedingly mucoid colonies on MSgg agar plates at $50^{\circ} \mathrm{C}$ (Fig. 5G) that were amorphous and highly extended in shape and size. This further supports the idea that PGA production is temperature-dependent. The mucosity was at a level such that when the petri dish plate was inverted the biomass dropped onto the lid. We observed similar phenotypes for colonies unable to produce EPS and surfactin (Fig. 5H, Fig. S3). We also found the mucoid phenotype was directly linked with PGA production as, contrary to the NCIB 3610 parental strain, the $p g s B$ deletion strains were entirely non-mucoid (Fig. 5I,J). We noted the $p g s B$ strain appeared to develop cell flares extending from the edge of the initial colony biofilm footprint, which would be consistent with secondary mutations evolving.

When the same strains were examined at $30^{\circ} \mathrm{C}$, no difference in the structure of the NCIB 3610 and pgsB colony biofilm architecture was observed (Fig. 5C,E), consistent with previous reports ${ }^{47,50}$. Similarly, both the eps $A-O$ and $p g s B$ epsA-O mutants are morphologically comparable (Fig. $5 \mathrm{D}, \mathrm{F}$ ). This, in conjunction with results presented in Fig. 4B-G, implies that the determining factor for colony morphology at low to intermediate temperature conditions is the EPS component of the biofilm matrix. Conversely, at high temperatures, PGA dominates as the extracellular component that controls biofilm structure. We found that the matrix mutants containing deletions in bslA, tasA, and epsA-O formed similarly mucoid biofilms at $50^{\circ} \mathrm{C}$ (Fig. S3). Only the mutant possessing a deletion in $\sin R$, while still mucoid, produced a biofilm with rugose structural features (Fig. S3). Collectively these data support the conclusion that PGA is produced in NCIB 3610 in both a density- and temperature-dependent manner and shows that biofilm architecture and structure can be dramatically influenced by the production of PGA. 


\section{Discussion}

\section{PGA production and motility}

In this work we have shown that B. subtilis produces PGA that, due to its humectant properties, induces a fluid flux into a growing colony. This up-welling of fluid induces cell motility that, in these high density and confined conditions, results in turbulent dynamics. It has been well established that motile and biofilm matrix-producing cell states are mutually exclusive ${ }^{26,28}$; any individual cell can be one but not both. It has also been demonstrated for both Gram-positive and Gram-negative species that active flagellated motility is often required for biofilm development and the role it plays is multi-fold ${ }^{51-53,53-56}$. It has been shown that the presence of motile cells can dramatically influence the rate of colony expansion when motile and non-motile cells are co-cultured ${ }^{57}$. Turbulent fluid flows generated by flagella-driven motility can drastically affect nutrient mixing ${ }^{58}$, transport of passive 'cargo' ${ }^{59}$, and may influence the formation of $B$. subtilis pellicle biofilms ${ }^{60}$. It is not yet clear what function PGAinduced motility may play in B. subtilis biofilm development. However, it is intriguing that that the same transcriptional regulators required for motility ${ }^{61}$ are also necessary for PGA synthesis ${ }^{47,62,63}$. In our experiments we observe motility in the same spatial location where fluid influx and, by inference, PGA production occurs. Whether the motility we observe is simply a by-product of being in a fluid environment, or whether there is a direct evolutionary and functional link between PGA production and motility is still an outstanding question.

From our experiments it is clear that high temperatures induce the colony to withdraw a considerable volume of fluid from the agar substrate. Previous work has shown that PGA can confer protection to bacteria and increase survival under many different environmental stresses ${ }^{64-67}$. It is plausible that when a biofilm is subject to temperature stress, an over-production of PGA into the extracellular environment would both scavenge and retain moisture which otherwise may be lost through evaporation, thus preventing desiccation of the colony. Additionally, a potential advantage of PGA production in a changing environment would be the possibility of active or passive spreading, to escape and search for more suitable environs to colonize.

\section{Spatial heterogeneity in matrix production}

Our experiments at intermediate temperatures $\left(38^{\circ} \mathrm{C}\right)$ are suggestive of spatial structuring between PGA-producing cells at the edge of the colony biofilm, and matrixproducing cells in the middle (as has previously been reported ${ }^{68}$ ), resulting in the annular confinement of fluid. Such spatial and temporal heterogeneity is a common feature in biofilms where the local microenvironment can strongly influence the phenotypic state of the cells. Phenotypic heterogeneity within a biofilm can be generated from variations in the chemical or physical environment ${ }^{69,70}$, genotypic variations, and stochastic gene expression ${ }^{69}$.

The heterogeneity in cell density imposed by the initial deposition conditions - and the formation of the 'coffee ring' - leads to the spatial pattern of fluid extraction that we observe. However, this is not the only means of generating density differences within a biofilm. Aggregates formed while growing in liquid culture can seed patches of higher cell density across the deposition footprint. Indeed, our results show that fluid invasion and, by inference, PGA production can occur in very local regions far away from the 'coffee ring' (Movie S4). This suggests that there is some critical density that 
determines whether a cell adopts a PGA producing state over a matrix-producing one.

The spatial heterogeneity is transient at $38^{\circ} \mathrm{C}$, and EPS becomes the dominant matrix component that determines the large-scale biofilm morphology. The fluid flux is ultimately stopped by the production of the EPS element of the matrix in the middle region of the biofilm (Fig. 6B). The motile cells that are close to the EPSproducing cells appear to stop moving, and a solid front advances from the middle of the colony outwards. One mechanism that could explain this abrupt transition involves the engagement of the "molecular clutch" EpsE, which binds to FliG and leads to motility arrest ${ }^{71}$. However, we may rule this scenario out since we observe the propagation of the motility arrest front in the epsA-O strain. It remains an open question of what physical or biological mechanism governs this phenomenon.

\section{Temperature dependent heterogeneity in matrix production}

At low temperatures we did not observe the phenotypic heterogeneity that we observe at intermediate temperatures, presumably due to a lack of PGA production (Fig. 6A) despite the high cell density in the coffee ring. At high temperatures, biofilms are extraordinarily mucoid and lack any discernible structure typical of a B. subtilis biofilm (Fig. 6C). In either extreme, the local heterogeneities seem to matter little and densitydependent PGA production is superseded by a temperature-dependent pathway which impacts the entire biofilm.

When engineered to overproduce the TasA fibres and exopolysaccharide, by introduction of a mutation in $\sin R$, the biofilms formed at high temperature (Fig. S3), were highly mucoid but also possessed structuring more typical of a wild-type $B$. subtilis biofilm. This implies that production of PGA can occur simultaneously with EPS/TasA, even if individual cells in the population commit to production of one or other product. This is also readily apparent at intermediate temperatures, where we infer that the two cell types have derived from the same population and are clearly present at the same time, albeit spatially separated.

Previous work has shown that strains possessing a deletion of spo0A result in biofilms that are mucoid and unstructured at low temperatures $\left(30^{\circ} \mathrm{C}\right)$ and that this was due to PGA production ${ }^{30,49}$. Therefore, a spoOA mutant broadly mimics the wild-type biofilm phenotype that we observe under high temperature conditions. This implies that Spo0A may be the regulatory component that controls temperature dependent production of a PGA-rich or EPS/TasA-rich biofilm matrix.

Taken together, these results imply that $B$. subtilis has the ability to adapt different biofilm matrices with distinct properties to fit disparate environmental conditions. It is plausible that such a strategy is employed in natural environments as $B$. subtilis can grow at a wide range of temperatures: it is found in desert soils and within composts which can easily reach temperatures of $50^{\circ} \mathrm{C}$. Until now, PGA was not thought to be an important factor as a matrix component in B. subtilis biofilms ${ }^{47}$. However, it appears, under the right conditions, PGA can become an alternative matrix component with distinct structural and physical characteristics that may aid the biofilm to survive in high temperature conditions.

\section{Physical Consequences of PGA Production}

The biofilm matrix of $B$. subtilis and $V$. cholerae has been modelled as a viscous hydrogel network that facilitates biofilm expansion via osmotic fluid influx ${ }^{72-75}$. The 
localization of EPS production at the propagating boundary of a growing colony is thought to drive the outward expansion of the biofilm. Concomitant production of osmolytes stimulates fluid extraction that swells the matrix at the growing boundary, driving motion forward. In our experiments, PGA seems to have the opposite effect: colony expansion stalls due to the colony entering a 'liquid-like' state when fluid is extracted from the substrate. Previous work has shown that colony biofilm expansion is strongly governed by mechanical contact forces between neighboring cells and friction with the underlying substrate ${ }^{57,76,77}$. In our experiments, expansion only recommences when the fluid environment dissipates, physical contacts are restored, and non-PGA matrix production begins. A question that remains is: how does colony expansion occur when PGA is the primary matrix component? Our experiments at high temperatures do show that the wild-type 3610 strain expands significantly beyond the initial deposition footprint. Strains that do not produce EPS or surfactin do not expand as much as the wild-type and this may hint that these components may still have a role to play in facilitating colony expansion (Fig. S3).

The traveling waves of fluid in the epsA-O deficient strain resulted in the appearance of finger-like structures as the wave propagated inwards. Such fingering instabilities can occur when a low viscosity fluid displaces one of a higher viscosity; the inverse situation will typically result in a stable interface. Curiously in our experiments, the fingers that we observe occur in the inverse configuration. Such inverse Saffman-Taylor instabilities can occur when there are wettable particles present that can adsorb to the air/fluid interface. This adsorption, due to interfacial energy minimization, can induce interfacial instabilities ${ }^{78}$. It is known that bacteria can accumulate at interfaces ${ }^{79}$, and B. subtilis can form floating biofilms at air/water interfaces. It is possible that bacteria coat and accumulate at the front of the incoming fluid wave, thus modifying the interfacial energetics and destabilising the interface between the fluid and the air at the interior of the colony. More work will need to be done to uncover the biological and physical mechanisms that cause this unusual phenomena and the possible benefit or function in ecological settings.

\section{Materials \& Methods}

\section{Growth conditions}

B. subtilis strains were initially grown in LB medium $(10 \mathrm{~g} \mathrm{NaCl}, 5 \mathrm{~g}$ yeast extract, and $10 \mathrm{~g}$ tryptone per liter). Biofilms were grown on MSgg agar (5 $\mathrm{mM}$ potassium phosphate and $100 \mathrm{mM}$ MOPs at pH 7.0 supplemented with $2 \mathrm{mM} \mathrm{MgCl}_{2}, 700 \mu \mathrm{M}$ $\mathrm{CaCl}_{2}, 50 \mu \mathrm{M} \mathrm{MnCl}_{2}, 50 \mu \mathrm{M} \mathrm{FeCl}_{3}, 1 \mu \mathrm{M} \mathrm{ZnCl}_{2}, 2 \mu \mathrm{M}$ thiamine, $0.5 \%$ v/v glycerol, $0.5 \% \mathrm{w} / \mathrm{v}$ glutamate, $1.5 \% \mathrm{w} / \mathrm{v}$ Select Agar (Invitrogen). When appropriate, antibiotics were used as required at the following concentrations: chloramphenicol at $5 \mu \mathrm{g}$ $\mathrm{ml}^{-1}$, kanamycin at $25 \mu \mathrm{g} \mathrm{ml}^{-1}$, spectinomycin at $100 \mu \mathrm{g} \mathrm{ml}{ }^{-1}$, and tetracycline at $10 \mu \mathrm{g} \mathrm{ml} l^{-1}$.

\section{Strain Construction}

All strains used in this study are provided in Table S1. All B. subtilis strains used in this work are derived from the wild-type laboratory isolate NCIB 3610 and constructed using standard protocols. SPP1 phage transduction was used for transfer of genomic DNA from the donor strain into the recipient NCIB 3610, as described previously ${ }^{80}$. 


\section{Biofilm Imaging and Analysis}

B. subtilis strains were inoculated into $3 \mathrm{~mL}$ LB from a single colony grown on $1.5 \%$ $\mathrm{w} / \mathrm{v}$ LB agar. The bacteria were allowed to grow at $30^{\circ} \mathrm{C}$ with $200 \mathrm{rpm}$ orbital shaking until reaching an OD between 1.5-2. The cell culture was diluted to OD 1.0 in phosphate-buffered saline. A $3 \mu \mathrm{L}$ droplet of bacteria was deposited onto a $35 \mathrm{~mm}$ petri dish (Corning) containing MSgg agar. The droplets of bacteria were allowed to dry for 10 minutes. After drying, the petri dish was placed on a Nikon Ti inverted microscope that is temperature controlled. All microscopy images and movies were captured using a CoolSNAP HQ2 CCD camera controlled by $\mu$ Manager software. Brightfield movies and images of the biofilms were captured from the underside of the petri dish and are imaged through the agar. For the bead tracking experiments, $1 \mu \mathrm{m}$ diameter latex carboxylate-modified polystyrene yellow fluorescent beads (Sigma-Aldrich) were diluted into PBS from the stock solution in a 1:1000 ratio. $1 \mu \mathrm{L}$ of the working solution was added to the $1 \mathrm{~mL}$ of the diluted cell culture just prior to deposition. Movies were acquired in bright and epifluorescent channels (Nikon GFP fluorescent filter cube) and the beads were tracked using the ImageJ plugin TrackMate (v3.8.0) ${ }^{81}$. All image analysis was performed using the Fiji distribution of ImageJ. Whole biofilm microscopy images were captured as above but in multiple tiles that were stitched together using the 'Pairwise Stitching' plug-in. The images of the fingering instabilities were generated by first using the 'straighten' tool to transform a circular to a linear region. The default ImageJ threshold method was applied to binarize the images. In cases where the intensity varied across the image, the image was partitioned into regions of similar intensity and then thresholding was performed. The 'edge finding' tool was used to locate the edges of the thresholded images. After edges were identified the interior was filled to form a representation of the fingers. If the image was partitioned, the image was stitched together using the stitching tool in ImageJ. Measurement of fluid travel distance was manually tracked in ImageJ and the mean displacement was averaged over 10 separate measurements for each experiment. For each strain studied, three separate experiments were performed. Edge and front displacement measurements were similarly performed in ImageJ by manually tracking the movement of the front over successive frames. Displacements were always measured relative to the initial position of each feature. Images of whole biofilms were obtained using a Leica MZ16 stereoscope as described previously ${ }^{17}$.

\section{Acknowledgements}

We thank M. Porter and F. Davidson for their helpful comments. Work in the NSW and CEM laboratories is funded by the Biotechnology and Biological Science Research Council (BBSRC) [BB/P001335/1, BB/R012415/1]. 
bioRxiv preprint doi: https://doi.org/10.1101/2020.12.21.423644; this version posted January 12, 2021. The copyright holder for this preprint (which was not certified by peer review) is the author/funder, who has granted bioRxiv a license to display the preprint in perpetuity. It is made available under aCC-BY-NC-ND 4.0 International license.

A

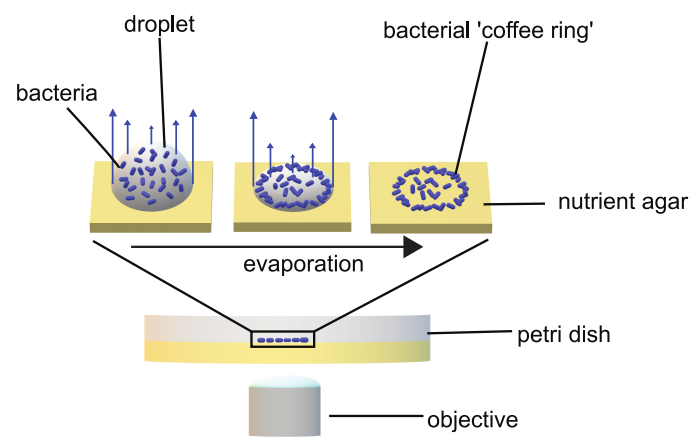

B
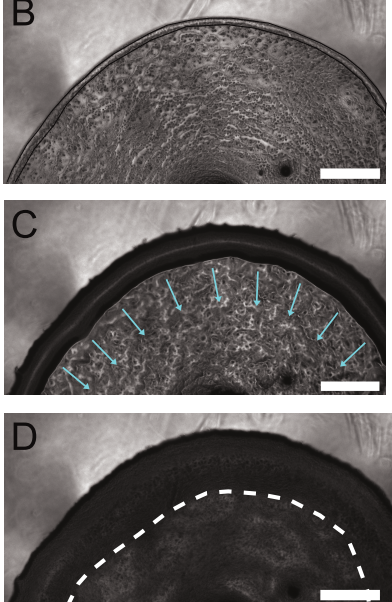
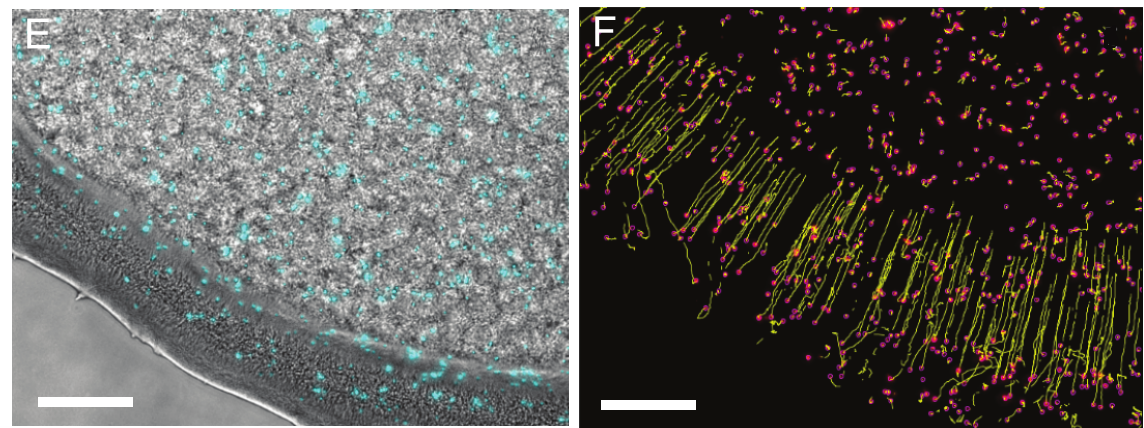

Figure 1. Schematic of the experiment and emergence of a travelling front of motile cells. (A) A $3 \mu \mathrm{L}$ droplet of a bacterial suspension is deposited onto the surface of MSgg agar and allowed to dry. Due to the differential rates of evaporation across the surface of the droplet (blue arrows), capillary flows are induced in the interior of the droplet. This allows for the transport of bacteria from the interior of the droplet to the edge where they become deposited. The effect is an accumulation of a higher density region of bacteria at the contact line between the droplet and solid agar. (B) This higher density region of bacteria can be seen at the edge of the colony. (C) After three hours of incubation and growth a darker region develops centered upon the edge of the emerging biofilm. We find that this zone is fluid and begins to move inwards towards the center of the colony (blue arrows). In this zone we observe active and motile cells. (D) After another two hours, the front stops moving inwards, indicated by the white dashed line, and the motility stops. Scale bars (B)-(D) are $500 \mu \mathrm{m}$. (E) Fluorescent beads were added to the suspension of bacteria and deposited with them at the beginning of the experiment; the beads are colored cyan. The 'motility zone' is identifiable as the darker region nearer the boundary of the colony (c.f. (C)). (F) Using particle tracking software, the beads are located (pink) and their motion tracked over time (yellow lines). The majority of beads move linearly at a constant rate towards the interior of the colony. Scale bars (E)-(F) $200 \mu \mathrm{m}$. 


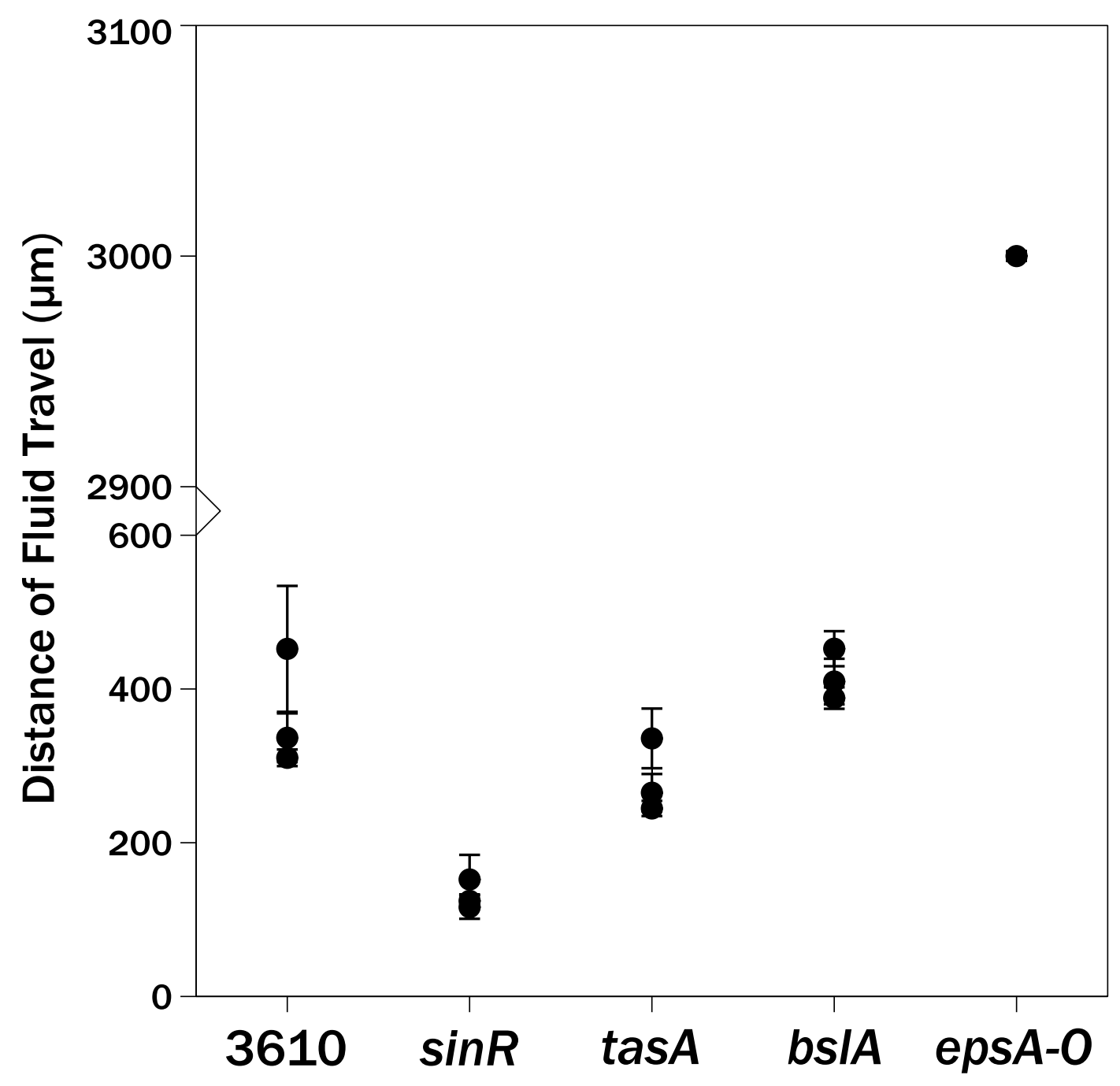

Figure 2. Matrix production can modify the spatial extent of fluid invasion. Plotted is the average distance of fluid travel as measured from the edge of the biofilm. The y-axis has been broken to show the differences between the $3610, \sin R, \operatorname{tas} A$, and $b s l A$ strains while still representing the large distance of fluid travel found in the epsA-O strain. Each data point is the mean fluid travel distance averaged over 10 spatial points across an individual biofilm ( $\mathrm{N}=3$ for each strain). Error bars represent standard deviation. 

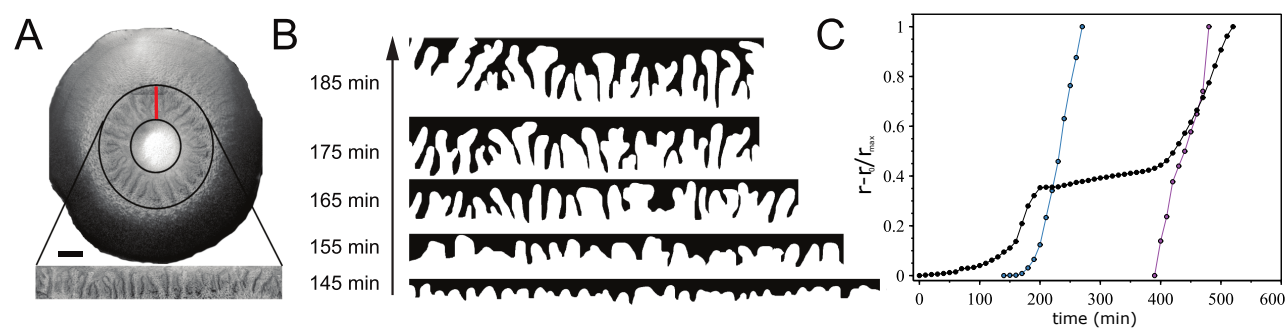

Figure 3. Large scale dynamics in polysaccharide-minus biofilms. (A) An example of a microscopy image of an entire epsA-O biofilm (scale bar is $500 \mu \mathrm{m}$ ). Annular black region defines the area where we observe finger-like instabilities. This annular region is made linear for ease of visualization; a cut is made at the red line and the circular region is 'unrolled' to form a linear region. (B) Binarized images of the fingers over time for the biofilm in (A). (C) The normalized displacement as a function of time for the outer edge of the colony (black), fluid front (blue) and motility arrest front (purple). A plateau in the outer edge growth occurs coincidentally with the onset of fluid propagation. The growth recommences after motility becomes arrested. The blue curve corresponds to the time when we observe the fingers develop in (A).
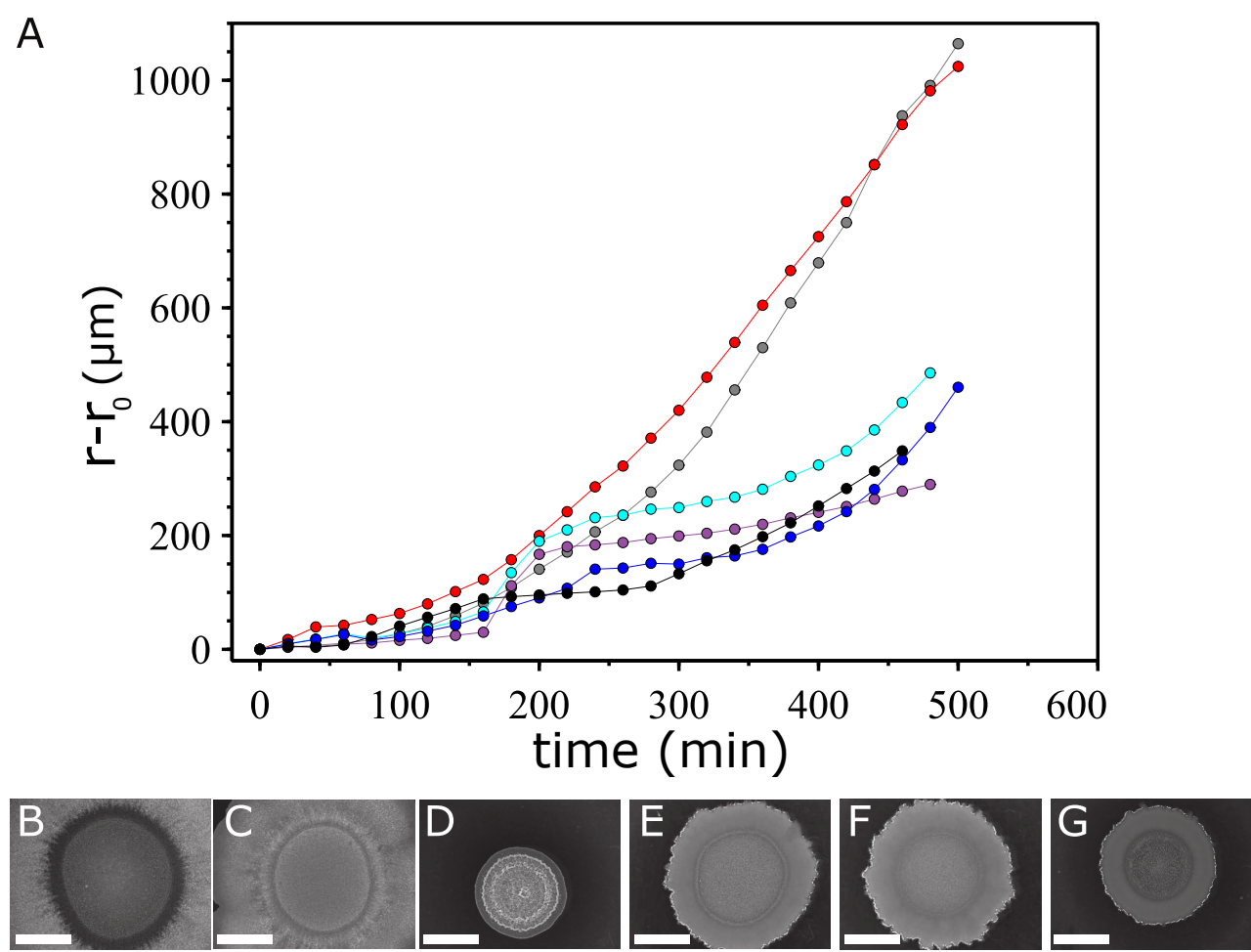

Figure 4. Fluid extraction is driven by PGA production. (A) Measurements of the outer edge displacement at $38^{\circ} \mathrm{C}$ show that NCIB 3610 (black), epsA-O (blue), the $\operatorname{srfAA}$ (purple), and srfAA epsA-O (cyan) strains possesses the characteristic 'fluidization plateau'. The pgs $B$ minus (red), and pgs $B$ eps $A-O$ minus strain (grey) that cannot produce PGA did not exhibit this behavior. Colony morphology after 48 hours incubation at $38^{\circ} \mathrm{C}$ of (B) NCIB 3610, (C) pgsB (D) srfAA (E) epsA-O, (F) pgsB epsA-O, and (G) srfAA epsA-O. Scale bar is 5 $\mathrm{mm}$. 

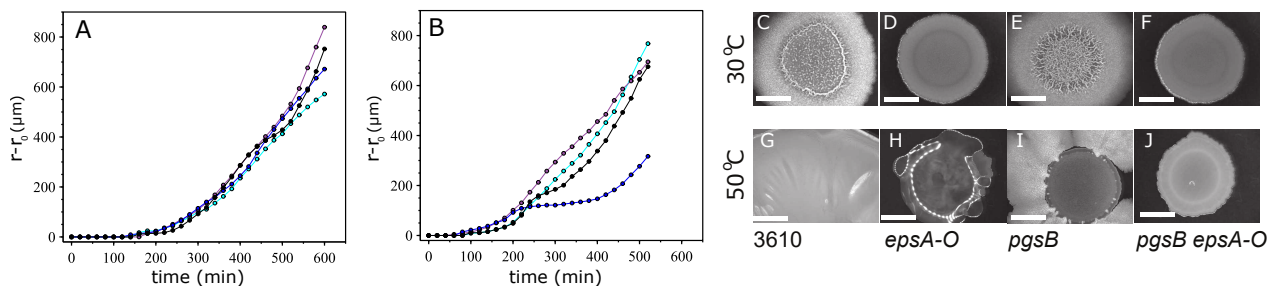

Figure 5. PGA production is temperature dependent. (A) Measurements of the outer edge displacement at $30^{\circ} \mathrm{C}$ and (B) $42^{\circ} \mathrm{C}$ for 3610 (black), epsA-O (blue), pgsB (magenta), pgsB epsA-O (cyan). Colony morphology after 48 hours incubation at $(\mathrm{C})-(\mathrm{F}) 30^{\circ} \mathrm{C}$, and $(\mathrm{G})-(\mathrm{J}) 50^{\circ} \mathrm{C}$. Scale bar is $5 \mathrm{~mm}$.

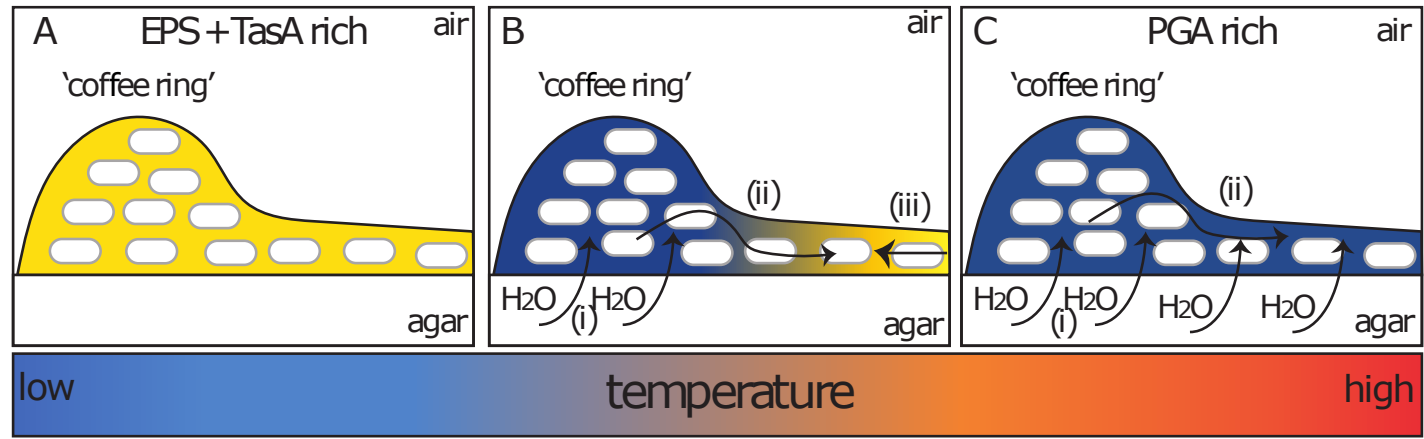

Figure 6. Schematic model of matrix production as a function of density and temperature. The 'coffee ring' is the initial region of higher density that is formed after deposition onto the agar surface. (A) Low temperatures produce a biofilm matrix rich in EPS and TasA (yellow). (B) Intermediate temperatures induces PGA production (blue) and (i) concomitant fluid extraction from the agar that originates in the high density region. (ii) The fluid propagates towards the center where (iii) EPS and TasA matrix production halts its advance. (C) High temperatures results in a PGA rich matrix that induces (i) fluid extraction that (ii) covers the entire biofilm resulting in a mucoid phenotype.

\section{References}

[1] Daniel B Kearns. Division of labour during Bacillus subtilis biofilm formation. Molecular Microbiology, 67(2):229-231, 2008.

[2] Daniel Kadouri and George A O'Toole. Susceptibility of biofilms to Bdellovibrio bacteriovirus attack. Applied and Environmental Microbiology, 71(7):4044-4051, 2005.

[3] Lucia Vidakovic, Praveen K Singh, Raimo Hartmann, Carey D Nadell, and Knut Drescher. Dynamic biofilm architecture confers individual and collective mechanisms of viral protection. Nature Microbiology, 3(1):26-31, 2018.

[4] Philip S Stewart and J William Costerton. Antibiotic resistance of bacteria in biofilms. The Lancet, 358(9276):135-138, 2001.

[5] CA Fux, J William Costerton, Philip S Stewart, and Paul Stoodley. Survival strategies of infectious biofilms. Trends in Microbiology, 13(1):34-40, 2005.

[6] J William Costerton, Philip S Stewart, and E Peter Greenberg. Bacterial biofilms: a common cause of persistent infections. Science, 284(5418):1318-1322, 1999.

[7] Barbara W Trautner and Rabih O Darouiche. Role of biofilm in catheter-associated urinary tract infection. American Journal of Infection Control, 32(3):177-183, 2004.

[8] Paul Stoodley, Dirk DeBeer, and Zbigniew Lewandowski. Liquid flow in biofilm systems. Applied and Environmental Microbiology, 60(8):2711-2716, 1994.

[9] Trevor Roger Garrett, Manmohan Bhakoo, and Zhibing Zhang. Bacterial adhesion and biofilms on surfaces. Progress in Natural Science, 18(9):1049-1056, 2008.

[10] Iwona B Beech and Jan Sunner. Biocorrosion: towards understanding interactions between biofilms and metals. Current Opinion in Biotechnology, 15(3):181-186, 2004.

[11] Rajbir Singh, Debarati Paul, and Rakesh K Jain. Biofilms: implications in bioremediation. 
Trends in Microbiology, 14(9):389-397, 2006.

[12] Anushree Malik. Metal bioremediation through growing cells. Environment International, $30(2): 261-278,2004$

[13] V Lazarova and J Manem. Biofilm characterization and activity analysis in water and wastewater treatment. Water Research, 29(10):2227-2245, 1995.

[14] Hanan Ivnitsky, Ilan Katz, Dror Minz, Galit Volvovic, Eyal Shimoni, Elina Kesselman, Raphael Semiat, and Carlos G Dosoretz. Bacterial community composition and structure of biofilms developing on nanofiltration membranes applied to wastewater treatment. Water Research, 41(17):3924-3935, 2007.

[15] Hera Vlamakis, Yunrong Chai, Pascale Beauregard, Richard Losick, and Roberto Kolter. Sticking together: building a biofilm the Bacillus subtilis way. Nature Reviews Microbiology, 11(3):157, 2013.

[16] Diego Romero, Claudio Aguilar, Richard Losick, and Roberto Kolter. Amyloid fibers provide structural integrity to Bacillus subtilis biofilms. Proceedings of the National Academy of Sciences USA, 107(5):2230-2234, 2010.

[17] Laura Hobley, Adam Ostrowski, Francesco V Rao, Keith M Bromley, Michael Porter, Alan R Prescott, Cait E MacPhee, Daan MF Van Aalten, and Nicola R Stanley-Wall. BslA is a self-assembling bacterial hydrophobin that coats the Bacillus subtilis biofilm. Proceedings of the National Academy of Sciences USA, 110(33):13600-13605, 2013.

[18] Keith M Bromley, Ryan J Morris, Laura Hobley, Giovanni Brandani, Rachel MC Gillespie, Matthew McCluskey, Ulrich Zachariae, Davide Marenduzzo, Nicola R Stanley-Wall, and Cait E MacPhee. Interfacial self-assembly of a bacterial hydrophobin. Proceedings of the National Academy of Sciences USA, 112(17):5419-5424, 2015.

[19] Kazuo Kobayashi and Megumi Iwano. Bsla (YuaB) forms a hydrophobic layer on the surface of Bacillus subtilis biofilms. Molecular Microbiology, 85(1):51-66, 2012.

[20] Massimiliano Marvasi, Pieter T Visscher, and Lilliam Casillas Martinez. Exopolymeric substances (EPS) from Bacillus subtilis: polymers and genes encoding their synthesis. FEMS Microbiology Letters, 313(1):1-9, 2010.

[21] Daniel T Verhamme, Ewan J Murray, and Nicola R Stanley-Wall. DegU and Spo0A jointly control transcription of two loci required for complex colony development by Bacillus subtilis. Journal of Bacteriology, 191(1):100-108, 2009.

[22] Sasha H Shafikhani, Ines Mandic-Mulec, Mark A Strauch, Issar Smith, and Terrance Leighton. Postexponential regulation of sin operon expression in Bacillus subtilis. Journal of Bacteriology, 184(2):564-571, 2002.

[23] Daniel B Kearns, Frances Chu, Steven S Branda, Roberto Kolter, and Richard Losick. A master regulator for biofilm formation by Bacillus subtilis. Molecular Microbiology, 55(3):739-749, 2005.

[24] U Bai, I Mandic-Mulec, and I Smith. SinI modulates the activity of SinR, a developmental switch protein of Bacillus subtilis, by protein-protein interaction. Genes $\&$ Development, 7(1):139-148, 1993.

[25] Kazuo Kobayashi. SlrR/SlrA controls the initiation of biofilm formation in Bacillus subtilis. Molecular Microbiology, 69(6):1399-1410, 2008.

[26] Yunrong Chai, Frances Chu, Roberto Kolter, and Richard Losick. Bistability and biofilm formation in Bacillus subtilis. Molecular Microbiology, 67(2):254-263, 2008.

[27] Jan Kampf, Jan Gerwig, Kerstin Kruse, Robert Cleverley, Miriam Dormeyer, Alexander Grünberger, Dietrich Kohlheyer, Fabian M Commichau, Richard J Lewis, and Jörg Stülke. Selective pressure for biofilm formation in Bacillus subtilis: differential effect of mutations in the master regulator SinR on bistability. mBio, 9(5):e01464-18, 2018.

[28] Daniel López and Roberto Kolter. Extracellular signals that define distinct and coexisting cell fates in Bacillus subtilis. FEMS Microbiology Reviews, 34(2):134-149, 2010.

[29] Yunrong Chai, Thomas Norman, Roberto Kolter, and Richard Losick. An epigenetic switch governing daughter cell separation in Bacillus subtilis. Genes 8 Development, 24(8):754-765, 2010.

[30] Steven S Branda, José Eduardo González-Pastor, Sigal Ben-Yehuda, Richard Losick, and 
Roberto Kolter. Fruiting body formation by Bacillus subtilis. Proceedings of the National Academy of Sciences USA, 98(20):11621-11626, 2001.

[31] Robert D Deegan, Olgica Bakajin, Todd F Dupont, Greb Huber, Sidney R Nagel, and Thomas A Witten. Capillary flow as the cause of ring stains from dried liquid drops. Nature, 389(6653):827-829, 1997.

[32] Jörn Dunkel, Sebastian Heidenreich, Knut Drescher, Henricus H Wensink, Markus Bär, and Raymond E Goldstein. Fluid dynamics of bacterial turbulence. Physical Review Letters, 110(22):228102, 2013.

[33] Tim Sanchez, Daniel TN Chen, Stephen J DeCamp, Michael Heymann, and Zvonimir Dogic. Spontaneous motion in hierarchically assembled active matter. Nature, 491(7424):431-434, 2012.

[34] George M Homsy. Viscous fingering in porous media. Annual Review of Fluid Mechanics, 19(1):271-311, 1987.

[35] SM Troian, XL Wu, and SA Safran. Fingering instability in thin wetting films. Physical Review Letters, 62(13):1496, 1989.

[36] A Hamraoui, M Cachile, C Poulard, and AM Cazabat. Fingering phenomena during spreading of surfactant solutions. Colloids and Surfaces A: Physicochemical and Engineering Aspects, 250(1-3):215-221, 2004.

[37] Magali Deleu, Hary Razafindralambo, Y Popineau, Philippe Jacques, Philippe Thonart, and Michel Paquot. Interfacial and emulsifying properties of lipopeptides from Bacillus subtilis. Colloids and Surfaces A: Physicochemical and Engineering Aspects, 152(1-2):3$10,1999$.

[38] Michiko M Nakano, MA Marahiel, and Peter Zuber. Identification of a genetic locus required for biosynthesis of the lipopeptide antibiotic surfactin in Bacillus subtilis. Journal of Bacteriology, 170(12):5662-5668, 1988.

[39] Daniel B Kearns. A field guide to bacterial swarming motility. Nature Reviews Microbiology, 8(9):634, 2010.

[40] Thomas E Angelini, Marcus Roper, Roberto Kolter, David A Weitz, and Michael P Brenner. Bacillus subtilis spreads by surfing on waves of surfactant. Proceedings of the National Academy of Sciences USA, 106(43):18109-13, Oct 2009.

[41] Daniel López, Hera Vlamakis, Richard Losick, and Roberto Kolter. Paracrine signaling in a bacterium. Genes \&f Development, 23(14):1631-1638, 2009.

[42] Marc Hennes, Julien Tailleur, Gaëlle Charron, and Adrian Daerr. Active depinning of bacterial droplets: The collective surfing of Bacillus subtilis. Proceedings of the National Academy of Sciences USA, 114(23):5958-5963, 2017.

[43] M Fata Moradali and Bernd HA Rehm. Bacterial biopolymers: from pathogenesis to advanced materials. Nature Reviews Microbiology, pages 1-16, 2020.

[44] Thomas Candela and Agnès Fouet. Poly-gamma-glutamate in bacteria. Molecular Microbiology, 60(5):1091-1098, 2006.

[45] Lung Shih and Yi-Tsong Van. The production of poly-( $\gamma$-glutamic acid) from microorganisms and its various applications. Bioresource Technology, 79(3):207-225, 2001.

[46] Zhiting Luo, Yuan Guo, Jidong Liu, Hua Qiu, Mouming Zhao, Wei Zou, and Shubo Li. Microbial synthesis of poly- $\gamma$-glutamic acid: current progress, challenges, and future perspectives. Biotechnology for Biofuels, 9(1):134, 2016.

[47] Nicola R Stanley and Beth A Lazazzera. Defining the genetic differences between wild and domestic strains of Bacillus subtilis that affect poly- $\gamma$-dl-glutamic acid production and biofilm formation. Molecular Microbiology, 57(4):1143-1158, 2005.

[48] Yi-Huang Hsueh, Kai-Yao Huang, Sikhumbuzo Charles Kunene, and Tzong-Yi Lee. Poly$\gamma$-glutamic acid synthesis, gene regulation, phylogenetic relationships, and role in fermentation. International Journal of Molecular Sciences, 18(12):2644, 2017.

[49] Yiyang Yu, Fang Yan, Yun Chen, Christopher Jin, Jian-Hua Guo, and Yunrong Chai. Poly- $\gamma$-glutamic acids contribute to biofilm formation and plant root colonization in selected environmental isolates of Bacillus subtilis. Frontiers in Microbiology, 7:1811, 2016.

[50] Steven S Branda, Frances Chu, Daniel B Kearns, Richard Losick, and Roberto Kolter. A 
major protein component of the Bacillus subtilis biofilm matrix. Molecular Microbiology, 59(4):1229-1238, 2006.

[51] George A O'Toole and Roberto Kolter. Flagellar and twitching motility are necessary for Pseudomonas aeruginosa biofilm development. Molecular Microbiology, 30(2):295-304, 1998.

[52] Paula I Watnick, Crystal M Lauriano, Karl E Klose, Laura Croal, and Roberto Kolter. The absence of a flagellum leads to altered colony morphology, biofilm development and virulence in Vibrio cholerae O139. Molecular Microbiology, 39(2):223-235, 2001.

[53] Katherine P Lemon, Darren E Higgins, and Roberto Kolter. Flagellar motility is critical for Listeria monocytogenes biofilm formation. Journal of Bacteriology, 189(12):4418-4424, 2007.

[54] Ali Houry, Romain Briandet, Stephane Aymerich, and Michel Gohar. Involvement of motility and flagella in Bacillus cereus biofilm formation. Microbiology, 156(4):1009-1018, 2010.

[55] Diego O. Serra, Anja M. Richter, Gisela Klauck, Franziska Mika, and Regine Hengge. Microanatomy at cellular resolution and spatial order of physiological differentiation in a bacterial biofilm. mBio, 4(2):e01464-18, 2013.

[56] Johanna Haiko and Benita Westerlund-Wikström. The role of the bacterial flagellum in adhesion and virulence. Biology, 2(4):1242-1267, 2013.

[57] Liyang Xiong, Yuansheng Cao, Robert Cooper, Wouter-Jan Rappel, Jeff Hasty, and Lev Tsimring. Flower-like patterns in multi-species bacterial colonies. eLife, 9:e48885, 2020.

[58] Jeffrey S Guasto, Roberto Rusconi, and Roman Stocker. Fluid mechanics of planktonic microorganisms. Annual Review of Fluid Mechanics, 44:373-400, 2012.

[59] Rasika M Harshey and Jonathan D Partridge. Shelter in a swarm. Journal of Molecular Biology, 427(23):3683-3694, 2015.

[60] Nitai Steinberg, Gili Rosenberg, Alona Keren-Paz, and Ilana Kolodkin-Gal. Collective vortex-like movement of Bacillus subtilis facilitates the generation of floating biofilms. Frontiers in Microbiology, 9:590, 2018.

[61] Cinzia Calvio, Cecilia Osera, Giuseppe Amati, and Alessandro Galizzi. Autoregulation of swrAA and motility in Bacillus subtilis. Journal of Bacteriology, 190(16):5720-5728, 2008.

[62] Taku Ohsawa, Kensuke Tsukahara, and Mitsuo Ogura. Bacillus subtilis response regulator $\mathrm{DegU}$ is a direct activator of $\mathrm{pgs} B$ transcription involved in $\gamma$-poly-glutamic acid synthesis. Bioscience, Biotechnology, and Biochemistry, 73(9):2096-2102, 2009.

[63] Cecilia Osera, Giuseppe Amati, Cinzia Calvio, and Alessandro Galizzi. SwrAA activates poly- $\gamma$-glutamate synthesis in addition to swarming in Bacillus subtilis. Microbiology, 155(7):2282-2287, 2009.

[64] AR Bhat, VU Irorere, T Bartlett, D Hill, G Kedia, Dimitris Charalampopoulos, S Nualkaekul, and I Radecka. Improving survival of probiotic bacteria using bacterial poly- $\gamma$ glutamic acid. International Journal of Food Microbiology, 196:24-31, 2015.

[65] Bao Tang, Dan Zhang, Sha Li, Zongqi Xu, Xiaohai Feng, and Hong Xu. Enhanced poly$\gamma$-glutamic acid production by $\mathrm{H}_{2} \mathrm{O}_{2}$-induced reactive oxygen species in the fermentation of Bacillus subtilis NX-2. Biotechnology and Applied Biochemistry, 63(5):625-632, 2016.

[66] Sy Le Thanh Nguyen, Takashi Inaoka, and Keitarou Kimura. Poly- $\gamma$-glutamic acid production by Bacillus subtilis (natto) under high salt conditions. Japan Agricultural Research Quarterly, 52(3):249-253, 2018.

[67] Kazuo Kobayashi. Plant methyl salicylate induces defense responses in the rhizobacterium Bacillus subtilis. Environmental Microbiology, 17(4):1365-1376, 2015.

[68] Siddarth Srinivasan, Ioana D Vladescu, Stephan A Koehler, Xiaoling Wang, Madhav Mani, and Shmuel M Rubinstein. Matrix production and sporulation in Bacillus subtilis biofilms localize to propagating wave fronts. Biophysical Journal, 114(6):1490-1498, 2018.

[69] Philip S Stewart and Michael J Franklin. Physiological heterogeneity in biofilms. Nature Reviews Microbiology, 6(3):199-210, 2008.

[70] Yijin Ren, Can Wang, Zhi Chen, Elaine Allan, Henny C van der Mei, and Henk J Busscher. 
Emergent heterogeneous microenvironments in biofilms: substratum surface heterogeneity and bacterial adhesion force-sensing. FEMS Microbiology Reviews, 42(3):259-272, 2018.

[71] Sarah B Guttenplan, Kris M Blair, and Daniel B Kearns. The epse flagellar clutch is bifunctional and synergizes with eps biosynthesis to promote Bacillus subtilis biofilm formation. PLoS Genet, 6(12):e1001243, 2010.

[72] Agnese Seminara, Thomas E Angelini, James N Wilking, Hera Vlamakis, Senan Ebrahim, Roberto Kolter, David A Weitz, and Michael P Brenner. Osmotic spreading of Bacillus subtilis biofilms driven by an extracellular matrix. Proceedings of the National Academy of Sciences USA, 109(4):1116-21, Jan 2012.

[73] Nir Ido, Amir Lybman, Shahar Hayet, David N Azulay, Mnar Ghrayeb, Sajeda Liddaweih, and Liraz Chai. Bacillus subtilis biofilms characterized as hydrogels. Insights on water uptake and water binding in biofilms. Soft Matter, 16:6180-6190, 2020.

[74] Siddarth Srinivasan, C Nadir Kaplan, and L Mahadevan. A multiphase theory for spreading microbial swarms and films. eLife, 8:e42697, 2019.

[75] Jing Yan, Carey D Nadell, Howard A Stone, Ned S Wingreen, and Bonnie L Bassler. Extracellular-matrix-mediated osmotic pressure drives Vibrio cholerae biofilm expansion and cheater exclusion. Nature Communications, 8(1):1-11, 2017.

[76] FDC Farrell, Oskar Hallatschek, D Marenduzzo, and B Waclaw. Mechanically driven growth of quasi-two-dimensional microbial colonies. Physical Review Letters, 111(16):168101, 2013.

[77] Dmitri Volfson, Scott Cookson, Jeff Hasty, and Lev S Tsimring. Biomechanical ordering of dense cell populations. Proceedings of the National Academy of Sciences USA, 105(40):15346-15351, 2008.

[78] Ilyesse Bihi, Michael Baudoin, Jason E Butler, Christine Faille, and Farzam Zoueshtiagh. Inverse Saffman-Taylor experiments with particles lead to capillarity driven fingering instabilities. Physical Review Letters, 117(3):034501, 2016.

[79] Liana Vaccari, Mehdi Molaei, Tagbo HR Niepa, Daeyeon Lee, Robert L Leheny, and Kathleen J Stebe. Films of bacteria at interfaces. Advances in Colloid and Interface Science, 247:561-572, 2017.

[80] Daniël T Verhamme, Taryn B Kiley, and Nicola R Stanley-Wall. DegU co-ordinates multicellular behaviour exhibited by Bacillus subtilis. Molecular Microbiology, 65(2):554568, 2007.

[81] Jean-Yves Tinevez, Nick Perry, Johannes Schindelin, Genevieve M Hoopes, Gregory D Reynolds, Emmanuel Laplantine, Sebastian Y Bednarek, Spencer L Shorte, and Kevin W Eliceiri. Trackmate: An open and extensible platform for single-particle tracking. Methods, 115:80-90, 2017. 